\title{
The isolated neutron star candidate 2XMM J104608.7-594306
}

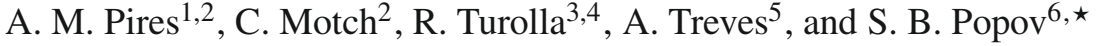 \\ 1 Instituto de Astronomia, Geofísica e Ciências Atmosféricas, Universidade de São Paulo, R. do Matão 1226 , \\ 05508-090 São Paulo, Brazil \\ e-mail: apires@astro.iag.usp.br \\ 2 CNRS, Université de Strasbourg, Observatoire Astronomique, 11 rue de l'Université, 67000 Strasbourg, France \\ 3 Universitá di Padova, Dipartimento di Fisica, via Marzolo 8, 35131 Padova, Italy \\ 4 Mullard Space Science Laboratory, University College London, Holmbury St. Mary, Dorking, Surrey, RH5 6NT, UK \\ 5 Universitá dell'Insubria, Dipartimento di Fisica e Matematica, Via Valleggio 11, 22100 Como, Italy \\ 6 Sternberg Astronomical Institute, Universitetskii pr. 13, 119991 Moscow, Russia
}

Received 15 September 2008 / Accepted 11 February 2009

\begin{abstract}
Over the last decade, X-ray observations have revealed the existence of several classes of isolated neutron stars (INSs) which are radio-quiet or exhibit radio emission with properties much at variance with those of ordinary radio pulsars. The identification of new sources is crucial in order to understand the relations among the different classes and to compare observational constraints with theoretical expectations. A recent analysis of the 2XMMp catalogue provided fewer than 30 new thermally emitting INS candidates. Among these, the source 2XMM J104608.7-594306 appears particularly interesting because of the softness of its X-ray spectrum, $k T=117 \pm 14 \mathrm{eV}$ and $N_{\mathrm{H}}=(3.5 \pm 1.1) \times 10^{21} \mathrm{~cm}^{-2}(3 \sigma)$, and of the present upper limits in the optical, $m_{\mathrm{B}} \gtrsim 26, m_{\mathrm{V}} \gtrsim 25.5$ and $m_{\mathrm{R}} \gtrsim 25$ (98.76\% confidence level), which imply a logarithmic X-ray-to-optical flux ratio $\log \left(F_{\mathrm{X}} / F_{\mathrm{V}}\right) \gtrsim 3.1$, corrected for absorption. We present the X-ray and optical properties of 2XMM J104608.7-594306 and discuss its nature in the light of two possible scenarios invoked to explain the X-ray thermal emission from INSs: the release of residual heat in a cooling neutron star, as in the seven radio-quiet ROSAT-discovered INSs, and accretion from the interstellar medium. We find that the present observational picture of 2XMM J104608.7-594306 is consistent with a distant cooling INS with properties in agreement with the most up-to-date expectations of population synthesis models: it is fainter, hotter and more absorbed than the seven ROSAT sources and possibly located in the Carina Nebula, a region likely to harbour unidentified cooling neutron stars. The accretion scenario, although not entirely ruled out by observations, would require a very slow $\left(\sim 10 \mathrm{~km} \mathrm{~s}^{-1}\right)$ INS accreting at the Bondi-Hoyle rate.
\end{abstract}

Key words. stars: neutron - X-rays: individuals: 2XMM J104608.7-594306 - catalogs

\section{Introduction}

A major outcome of the ROSAT mission has been the discovery of a group of radio-quiet isolated neutron stars (INSs) with properties clearly at variance with the bulk of the population of rotation-powered pulsars. At present, the group comprises seven objects (whence the nickname "Magnificent Seven", or M7) sharing similar properties, which include purely thermal X-ray spectra with very soft temperatures $(k T \sim 40-100 \mathrm{eV})$ and low absorption column densities $\left(N_{\mathrm{H}} \sim\right.$ few $\left.10^{20} \mathrm{~cm}^{-2}\right)$, long spin periods $(P \sim 3-10 \mathrm{~s})$, intense magnetic fields $\left(B \sim 10^{13}-10^{14} \mathrm{G}\right)$, very faint optical counterparts $\left(m_{\mathrm{B}} \gtrsim 25\right.$ when detected, implying X-ray-to-optical flux ratios $F_{\mathrm{X}} / F_{\text {opt }} \gtrsim 10^{4}$ ) and no associations with supernova remnants or stellar companions (see e.g. Haberl 2007, for a recent review).

For some time, following the pioneering work by Ostriker et al. (1970) and Shvartsman (1971), it was thought that these

\footnotetext{
* Based on the public data archive of XMM-Newton an ESA Science Mission with instruments and contributions directly funded by the ESA Member States and the USA (NASA) and a Chandra Legacy programme. Optical observations were performed at the European Southern Observatory, Paranal, Chile, under programme ID 079.D0633(A), and at the Southern Astrophysical Research Telescope, Cerro Pachón, Chile.
}

sources might belong to the long sought population of old INSs powered by accretion of the interstellar medium (ISM), which was expected to be detected by ROSAT (see Treves et al. 2000, for a review). However, the measurement of large proper motions (e.g. Motch et al. 2007, 2009, and references therein) strongly supports the idea that the M7 represent a local population ( $d \lesssim 500$ pc, Posselt et al. 2007; van Kerkwijk \& Kaplan $2007)$ of middle-aged neutron stars $\left(\sim 10^{5}-10^{6} \mathrm{yr}\right)$, which give off thermal radiation as they cool down. The similar spin periods and their position in the $P-\dot{P}$ diagram raised the possibility that they could be related to other populations of INSs - like magnetars, high magnetic field radio pulsars (HBPSRs) and rotating radio transients (RRATs; McLaughlin et al. 2006) - which is an intriguing and still debated issue (e.g. Popov et al. 2006; Kaplan 2008; Keane \& Kramer 2008).

Considering that within $1 \mathrm{kpc}$ the M7 appear in comparable numbers as young ( $\lesssim$ few Myr) radio and $\gamma$-ray pulsars (Popov et al. 2003), they may represent the only identified members of a large, yet undetected, elusive population of radio-quiet and thermally emitting INSs. The discovery of new candidates is then mandatory in order to make any progress towards an understanding of their properties as a population and of their relation to other classes of Galactic INSs. 
Despite the relatively small field-of-view and low sky coverage of the XMM-Newton Observatory ${ }^{1}$, its large effective area and good positional accuracy at soft X-ray energies make it ideal to look for faint INS candidates. Recently, Pires \& Motch (2008) reported on the preliminary results of a programme aimed at identifying new thermally emitting INSs in the 2XMMp catalogue $^{2}$. This version of the catalogue, released in July 2006, contains more than 120 thousand sources, covering a total sky area of $\sim 285 \mathrm{deg}^{2}$. Out of the considered $\sim 7.2 \times 10^{4}$ EPIC pn sources with count rates above $0.01 \mathrm{~s}^{-1}$, fewer than 30 good candidates met all the selection criteria - i.e. well detected pointlike sources showing a soft thermal spectrum $(k T \leq 200 \mathrm{eV}$ and $N_{\mathrm{H}}=10^{19}-10^{22} \mathrm{~cm}^{-2}$ ), with no optical candidates and no identifications in over 170 astronomical catalogues. The brightest and most promising INS candidate is source 2XMM J104608.7594306 (hereafter XMM J1046). In this work we discuss the properties of XMM J1046 in connection with the two main options concerning its nature, a cooling or an accreting INS. The description of the selection procedure and the results of followup optical observations on a sample of the X-ray brightest INS candidates will be presented elsewhere (Pires et al. 2009, submitted).

\section{XMM J1046 in X-rays and the optical}

\subsection{Observations and data reduction}

Thanks to the fact that source XMM J1046 is at an angular distance of only $\sim 8.5$ arcmin from the well studied binary system Eta Carinae, it was serendipitously observed by the EPIC pn and MOS detectors on board XMM-Newton on many different occasions. As part of an observational campaign to study the star-forming region of the Carina Nebula, XMM J1046 was also serendipitously observed once in a recent Chandra observation (September 2008). Although this X-ray mission observed Eta Carinae many times, XMM J1046 was not detected before given the smaller field-of-view of the ACIS instruments relative to the EPIC cameras.

We analysed 16 XMM-Newton archival observations, spanning from July 2000 to February 2006, in which XMM J1046 was visible. The event files were processed applying standard procedures with SAS 7.1.0 $0^{3}$. The MOS and pn observations were reduced using emchain and epchain, respectively, applying default corrections. The event lists were filtered for intervals of high background activity as well as to retain the pre-defined patterns corresponding to single, double, triple and quadruple pixel events for the MOS observations, and single and double pixel events for the pn observations, as these have the best energy calibration. Source and background events were extracted using circular regions of radii $\sim 25$ arcsec (centered on the position of the X-ray source) and $\sim 50$ arcsec, respectively. Background regions were defined on an area free of sources in the same CCD and roughly at the same distance from the readout node as the source region. We restricted our analysis to the $0.15-3 \mathrm{keV}$ energy range. Whenever possible for a given observation, data from all EPIC cameras were analysed simultaneously to better constrain the spectral parameters.

\footnotetext{
1 In the near future, eROSITA will become an excellent tool to search for INSs (http: //WWW .mpe.mpg.de/projects.html\#erosita).

2 http://xmmssc-www.star.le.ac.uk/Catalogue/

xcat_public_2XMMp.html

3 http://xmm.esac.esa.int/sas
}

The Chandra observation was analysed using CIAO 4.0.1 and CALDB 3.4.5 ${ }^{4}$. We processed the event files with the task acis_process_events, also applying default corrections. Since the observation was taken in VFAINT mode, we cleaned the ACIS background while processing the event file. We checked for the presence of known processing offsets using the aspect calculator tool available on the CIAO web pages. Finally, we selected events corresponding to grades 0, 2, 3, 4 and 6 (in ASCA terminology) and applied the good time intervals, also filtering for periods of background flares. For the spectral analysis, we used the $0.5-3 \mathrm{keV}$ energy band due to the molecular contamination that degrades the quantum efficiency of the ACIS frontilluminated chips, at energies below $0.5 \mathrm{keV}^{5}$. Source and background regions were extracted applying similar criteria as for $X M M$-Newton data. In Table 1 we list, for each observation, the effective exposure times, off-axis angles, the number of extracted source photons for the spectral analysis and the source count rate, corrected for vignetting, in the given energy bands.

\subsection{Spectral analysis}

EPIC and Chandra images do not reveal any particular background enhancement close to the X-ray source. However, the Chandra data, which benefit from a lower instrumental background, do show some weak diffuse extended emission (a filamentary structure roughly consistent with some large-scale nebulosity seen in the optical ${ }^{6}$ ). The brightest part is located 5 to 7 arcmin south-east of the X-ray source. This filament is not detected on the XMM-Newton images, although it is seen marginally in the field-of-view of the EPIC cameras. None of the background regions used in the spectral analysis overlaps with this diffuse X-ray emission. Therefore, it is expected that the noise for the EPIC data is dominated by the (position independent) instrumental background. The noticeable vignetting and spreading of the PSF prevailing at the large off-axis angles where the source lies in many XMM-Newton observations both contribute to the difficulty in measuring the spectrum of XMM J1046 in these cases.

Spectra were binned requiring a different minimum number of counts per energy bin, depending on the total number of source counts. Spectra extracted from the shortest exposures have at least 5 counts per bin. Using XSPEC 12.47, we tested different models (blackbody, power-law, bremsstrahlung, Raymond-Smith, ...), allowing the fit parameters to vary freely. Due to the low number of counts, we applied the maximum likelihood $C$ statistic (Cash 1979) in order to derive the best fit parameters and their uncertainties. The quality of each fit ("goodness") is estimated by means of Monte Carlo simulated spectra, drawn from the best fit model, and the distribution of corresponding $C$ fit statistics. If most $(>50 \%)$ of the simulated spectra have a smaller fit statistic than the current model, then it is unlikely that the observed data were drawn from the model.

The spectrum of XMM J1046 is always best fitted by a single absorbed soft blackbody; other models (in particular, powerlaw, optically thin thermal plasmas and magnetized neutron star atmospheres) invariably result in worse fits and there is no evidence for an additional (hard) component. In general, the X-ray emission of radio pulsars results from the sum of thermal and

\footnotetext{
4 http://cxc.harvard.edu/ciao4.0/index.html

5 http://cxc.harvard. edu/proposer/POG

6 ESO MAMA-R digitized plate. This filament is not in the field-ofview of our ESO/SOAR images.

7 http://heasarc.gsfc.nasa.gov/docs/xanadu/xspec
} 
Table 1. Description of the set of XMM-Newton and Chandra observations that serendipitously detected source XMM J1046.

\begin{tabular}{|c|c|c|c|c|c|c|c|c|c|}
\hline$\overline{\text { OBSID }}$ & Observation Date & Detector & Filter & $\begin{array}{l}t_{\text {exp }} \\
\text { (ks) }\end{array}$ & $\begin{array}{c}\theta \theta \\
\text { (arcmin) }\end{array}$ & $\begin{array}{l}t_{\text {eff }} \\
(\mathrm{ks})\end{array}$ & Counts & $\begin{array}{l}\text { Rate } \\
\left(10^{-2} \mathrm{~s}^{-1}\right)\end{array}$ & Near Gap? \\
\hline \multirow[t]{3}{*}{112580601} & $2000-07-26$ & pn & thick & 27.7 & 9.7 & 15.0 & 558 & $3.73 \pm 0.18$ & yes \\
\hline & & M1 & thick & 33.2 & 8.7 & 17.8 & 244 & $1.37 \pm 0.10$ & no \\
\hline & & M2 & thick & 30.1 & 9.1 & 15.7 & 198 & $1.26 \pm 0.10$ & yes \\
\hline \multirow[t]{3}{*}{112580701} & $2000-07-27$ & pn & thick & 8.0 & 9.7 & 4.3 & 161 & $3.7 \pm 0.3$ & yes \\
\hline & & M1 & thick & 10.9 & 8.7 & 5.9 & 88 & $1.50 \pm 0.19$ & no \\
\hline & & M2 & thick & 7.9 & 9.1 & 4.1 & 41 & $1.00 \pm 0.18$ & yes \\
\hline 112560101 & $2001-06-25$ & pn & thick & 21.5 & 15.8 & 6.7 & 291 & $4.4 \pm 0.3$ & yes \\
\hline 112560201 & 2001-06-28 & pn & thick & 20.5 & 15.9 & 6.3 & 200 & $3.2 \pm 0.3$ & yes \\
\hline 145740101 & $2003-01-25$ & M1 & thick & 6.9 & 8.3 & 4.6 & 78 & $1.68 \pm 0.22$ & yes \\
\hline \multirow[t]{2}{*}{145740201} & 2003-01-27 & M1 & thick & 6.9 & 8.3 & 4.6 & 73 & $1.59 \pm 0.21$ & yes \\
\hline & & M2 & thick & 6.9 & 8.0 & 4.8 & 69 & $1.44 \pm 0.20$ & yes \\
\hline \multirow{2}{*}{145740301} & $2003-01-27$ & M1 & thick & 6.8 & 8.3 & 4.5 & 59 & $1.30 \pm 0.20$ & yes \\
\hline & & M2 & thick & 6.8 & 8.0 & 4.7 & 68 & $1.44 \pm 0.19$ & yes \\
\hline 145740501 & 2003-01-29 & M2 & thick & 6.9 & 7.9 & 4.8 & 67 & $1.41 \pm 0.19$ & yes \\
\hline \multirow[t]{2}{*}{160160101} & 2003-06-08 & M1 & thick & 16.5 & 8.5 & 8.8 & 127 & $1.44 \pm 0.15$ & yes \\
\hline & & M2 & thick & 15.5 & 8.2 & 8.6 & 114 & $1.33 \pm 0.15$ & no \\
\hline \multirow[t]{2}{*}{160160901} & 2003-06-13 & M1 & thick & 31.1 & 8.5 & 16.6 & 207 & $1.25 \pm 0.10$ & no \\
\hline & & M2 & thick & 31.1 & 8.3 & 17.1 & 217 & $1.27 \pm 0.10$ & no \\
\hline 145780101 & $2003-07-22$ & M1 & thick & 8.4 & 9.4 & 4.6 & 76 & $1.66 \pm 0.21$ & no \\
\hline 160560101 & 2003-08-02 & M2 & medium & 11.8 & 9.1 & 6.3 & 121 & $1.93 \pm 0.19$ & no \\
\hline \multirow[t]{2}{*}{160560201} & 2003-08-09 & M1 & thick & 12.2 & 8.6 & 6.8 & 105 & $1.54 \pm 0.17$ & no \\
\hline & & M2 & medium & 12.1 & 9.1 & 6.4 & 96 & $1.50 \pm 0.18$ & no \\
\hline \multirow[t]{2}{*}{160560301} & 2003-08-18 & M1 & thick & 18.5 & 8.6 & 10.8 & 155 & $1.42 \pm 0.13$ & no \\
\hline & & M2 & medium & 18.5 & 9.4 & 10.0 & 160 & $1.60 \pm 0.14$ & no \\
\hline 206010101 & 2004-12-07 & pn & medium & 19.3 & 17.3 & 5.8 & 366 & $6.3 \pm 0.4$ & no \\
\hline \multirow[t]{2}{*}{311990101} & 2006-01-31 & pn & thick & 24.3 & 7.7 & 15.9 & 837 & $5.27 \pm 0.26$ & no \\
\hline & & M2 & thick & 65.3 & 8.2 & 44.2 & 731 & $1.65 \pm 0.15$ & no \\
\hline 9488 & 2008-09-05 & ACIS-I & $\mathrm{OBF}$ & 60.0 & 6.7 & 57.0 & 567 & $0.95 \pm 0.04$ & no \\
\hline
\end{tabular}

Note: the exposure times $\left(t_{\exp }\right)$, filtered for background flares, and the effective exposures $\left(t_{\mathrm{eff}}\right)$ on XMM J1046, accounting for vignetting, are reported; $\theta$ is the source off-axis angle. Net source photons and the on-axis count rates are in the $0.15-3 \mathrm{keV}$ (XMM-Newton) and $0.5-3 \mathrm{keV}$ (Chandra) energy bands.

non-thermal components (see e.g. Kaspi et al. 2004, for a review). A power-law component usually dominates the X-ray emission of young pulsars (age $\lesssim 10^{5} \mathrm{yr}$ ) while old pulsars (age zfew Myr) exhibit a weak thermal component, probably originating from small heated polar caps, in addition to a dominating power-law. On the other hand, the X-ray emission of the middleaged pulsars (age $\sim$ few $10^{5}$ yr) known as "Three Musketeers" is clearly dominated by soft blackbody components superposed on a high energy tail with photon indexes 1.7-2.1 (e.g. De Luca et al. 2005). The addition to the blackbody model of a powerlaw component with similar photon indexes does not improve the fit of XMM J1046. Any power-law component contributes at most $4 \%$ ( $3 \sigma$ confidence level, $0.5-10 \mathrm{keV}$ range) to the source luminosity. Therefore, a power-law flux as low, relative to the thermal component, as that of the Three Musketeers $(0.3-1.7 \%)$ would not be detectable in XMM J1046 with the available data and presently cannot be excluded. However, an X-ray spectrum dominated by a non-thermal component is clearly ruled out.

The M7 show rather stable spectral and timing properties. In particular, the X-ray brightest source RX J1856.5-3754 shows very constant flux and spectral properties over several years. Its $\mathrm{X}$-ray spectrum is remarkably well reproduced by a blackbody with no significant deviations (e.g. Burwitz et al. 2003). On the other hand, the second brightest source RX J0720.4-3125 is the only one among the M7 that has been shown to undergo long term variation in its spectral parameters, at more or less constant flux. This behaviour is possibly either cyclic and related to the star precession (Haberl et al. 2006; Hohle et al. 2009, and references therein) or impulsive, being interpreted as a sudden change on the neutron star surface accompanied with a simultaneous torque, caused by an accretion or glitch episode (van Kerkwijk et al. 2007).

Considering the 16 XMM-Newton observations, single absorbed blackbodies have temperatures and column densities ${ }^{8}$ ranging from $76_{-11}^{+18}$ to $160_{-23}^{+28} \mathrm{eV}$ and from $\left(1.4_{-0.9}^{+1.1}\right) \times 10^{21}$ to $\left(8.5_{-2.8}^{+2.6}\right) \times 10^{21} \mathrm{~cm}^{-2}$; errors are $1 \sigma$. In some cases, the small number of counts prevents a well constrained fit. In order to check the significance of the variations of the spectral parameters of XMM J1046, we performed a $\chi^{2}$ test on the 16 XMM-Newton observations, assuming that they do not change in time and have values equal to their respective weighted means $k T=$ $113 \pm 11 \mathrm{eV}$ and $N_{\mathrm{H}}=(3.6 \pm 0.9) \times 10^{21} \mathrm{~cm}^{-2}$ (hereafter, reported errors on the weighted means are $3 \sigma$, unless otherwise noted). We found that $k T$ and $N_{\mathrm{H}}$ are consistent with being the same in all observations at an acceptable but low confidence level for the temperature $\left(\chi_{k T}^{2} \sim 26\right.$ and $\chi_{N_{\mathrm{H}}}^{2} \sim 19$ for 15 degrees of freedom, respectively; the probabilities of obtaining a larger $\chi^{2}$ are $\sim 4 \%$ and $\sim 19 \%$ ). However, we note that among the data sets with the largest values of $\chi^{2}$ there are some observations for which the source elongated point spread function (PSF) overlaps the CCD gaps of the EPIC instruments. The correction for the missing part of the energy-dependent PSF might not be well calibrated, in particular at large off-axis angles (XMM J1046 is located at $\sim 9$ arcmin, on average, but its off-axis angle can be as large as $\sim 17$ arcmin, see Table 1). This would require the inclusion

\footnotetext{
8 The errors on $N_{\mathrm{H}}$ reported in Pires \& Motch (2008, Table 2) should be multiplied by a factor of 10 .
} 
Table 2. X-ray spectral analysis of INS candidate XMM J1046.

\begin{tabular}{|c|c|c|c|c|c|c|c|c|c|c|c|}
\hline \multirow[b]{2}{*}{ OBSID } & \multirow[b]{2}{*}{ Detector } & \multicolumn{6}{|c|}{ Absorbed blackbody fit } & \multicolumn{4}{|c|}{ Constant $N_{\mathrm{H}}$} \\
\hline & & $\begin{array}{c}N_{\mathrm{H}} \\
\left(10^{21} \mathrm{~cm}^{-2}\right)\end{array}$ & $\begin{array}{c}k T \\
(\mathrm{eV})\end{array}$ & $\begin{array}{c}f_{\mathrm{X}, 14} \\
\left(\mathrm{erg} \mathrm{s}^{-1} \mathrm{~cm}^{-2}\right)\end{array}$ & $C$ & d.o.f. & $\begin{array}{c}\text { Goodness } \\
(\%)\end{array}$ & $\begin{array}{c}k T \\
(\mathrm{eV})\end{array}$ & $C$ & d.o.f. & $\begin{array}{c}\text { Goodness } \\
(\%)\end{array}$ \\
\hline 112580601 & M1 & $3.7_{-1.1}^{+1.4}$ & $132_{-17}^{+16}$ & $10.4(8)$ & 19 & 21 & 43.7 & $134(5)$ & 19 & 20 & 40.6 \\
\hline 112580701 & M1 & $3.8_{-2.1}^{+2.1}$ & $124_{-23}^{+35}$ & $11.6_{-1.4}^{+1.5}$ & 15 & 21 & 11.2 & $128_{-8}^{+9}$ & 15 & 20 & 12.6 \\
\hline 160160101 & M2 & $5.6_{-2.3}^{+3}$ & $103_{-21}^{+26}$ & $9.9_{-1.0}^{+1.1}$ & 19 & 13 & 81.6 & $125_{-6}^{+7}$ & 20 & 12 & 87.2 \\
\hline 160160901 & $\mathrm{M} 1 / \mathrm{M} 2$ & $3.1_{-1.0}^{+0.8}$ & $132_{-12}^{+17}$ & $10.3(6)$ & 25 & 32 & 13.1 & $126(4)$ & 25 & 31 & 16.0 \\
\hline 145780101 & M1 & $2.7_{-2.0}^{+2.0}$ & $124_{-26}^{+12}$ & $12.9_{-1.6}^{+1.7}$ & 15 & 24 & 3.6 & $114_{-7}^{+8}$ & 16 & 23 & 4.0 \\
\hline 160560101 & M2 & $5.9_{-4}^{+2.6}$ & $96_{-23}^{+45}$ & $11.5(1.2)$ & 34 & 37 & 25.0 & $121_{-6}^{+7}$ & 35 & 36 & 25.7 \\
\hline 160560201 & $\mathrm{M} 1 / \mathrm{M} 2$ & $1.4_{-0.9}^{-4.1}$ & $160_{-23}^{+23}$ & $11.6(9)$ & 53 & 42 & 79.5 & $122(5)$ & 56 & 41 & 88.8 \\
\hline 160560301 & M1/M2 & $6.0_{-1.1}^{+2.3}$ & $92_{-14}^{+9}$ & $10.5_{-0.8}^{+0.7}$ & 33 & 30 & 44.9 & $115_{-3}^{+4}$ & 37 & 32 & 65.9 \\
\hline 206010101 & pn & $4.0_{-1.0}^{+1.1}$ & $106_{-12}^{+14}$ & $9.0(6)$ & 35 & 26 & 86.7 & $112(4)$ & 35 & 25 & 89.1 \\
\hline 311990101 & $\mathrm{pn} / \mathrm{M} 2$ & $3.9_{-0.5}^{+0.0}$ & $122(8)$ & $10.1(3)$ & 62 & 65 & 38.1 & $127(2)$ & 63 & 64 & 43.0 \\
\hline 9488 & ACIS-I & $6.3_{-1.2}^{+0.7}$ & $103_{-8}^{+9}$ & $7.8(3)$ & 13 & 20 & 12.9 & $125_{-3}^{+4}$ & 18 & 18 & 37.7 \\
\hline
\end{tabular}

Note: Only the set of 10 best XMM-Newton observations (see text) and the Chandra one are shown. The best fit parameters (derived using the maximum likelihood $C$ statistic) listed are for an absorbed blackbody model for which all parameters were allowed to vary freely (middle column). The right column shows the blackbody temperatures obtained when the column density is held constant at the mean value, $N_{\mathrm{H}}=3.5 \times 10^{21} \mathrm{~cm}^{-2}$. Errors are $1 \sigma$. The observed flux $f_{\mathrm{X}, 14}$ refers to ranges $0.15-3 \mathrm{keV}$ and $0.5-3 \mathrm{keV}$ for XMM-Newton and Chandra data, respectively, in units of $10^{-14} \mathrm{erg} \mathrm{s}^{-1} \mathrm{~cm}^{-2}$. The "goodness-of-fit" is derived from 1000 Monte Carlo simulated spectra.

of an additional systematic error which would have the effect of lowering the $\chi^{2}$ values. On the other hand, if we only consider the 10 observations for which the source is not located close to a gap ${ }^{9}$, we find that the temperature and column density are steady over the six-year time interval at rather high confidence levels: $k T=117 \pm 14 \mathrm{eV}(23 \%)$ and $N_{\mathrm{H}}=(3.5 \pm 1.1) \times 10^{21} \mathrm{~cm}^{-2}(44 \%)$, see Table 2 .

Alternatively, if the column density is held constant at the mean value $N_{\mathrm{H}}=3.5 \times 10^{21} \mathrm{~cm}^{-2}$, the computed range in $k T$ is narrower, $111-132 \mathrm{eV}$, and the parameters are better constrained ( $1 \sigma$ errors correspond to $\sim 5 \%$ of the best values against $\sim 20 \%$ when $N_{\mathrm{H}}$ is free to vary, see Table 2 for the individual fits). In this case, the variations in $k T$ are statistically significant $(99.26 \%)$. We note, however, that the sample of analysed observations is highly heterogeneous and thus subject to systematic uncertainties. In particular, the different observing conditions - distance to the optical axis, observing modes and cameras - as well as calibration uncertainties at energies below $0.5 \mathrm{keV}$ especially for the MOS cameras ${ }^{10}$, do not allow one to draw definite conclusions on the source intrinsic spectral variability. Hereafter, we adopt the weighted mean blackbody temperature and column density of the 10 best XMM-Newton observations for the purpose of further discussion on the source properties. In Fig. 1 the individual best fits are plotted together with the $N_{\mathrm{H}} \times k T$ contours obtained fitting all data with one single blackbody model.

A slightly more absorbed blackbody fit is found for the Chandra observation (Table 2). We note, however, that the response of the front-illuminated ACIS chips is not best suited to derive the spectral parameters of a source as soft as XMM J1046. This, and the fact that only photons with energies above $0.5 \mathrm{keV}$ were considered, act together to cast some doubts on the column density derived in the blackbody fit. In Fig. 2 we show the spectral fits of the two data sets with the best signal-to-noise ratio among the analysed data.

The weighted mean of the $0.15-3 \mathrm{keV}$ observed flux is $f_{\mathrm{X}}=$ $(9.7 \pm 0.5) \times 10^{-14} \mathrm{erg} \mathrm{s}^{-1} \mathrm{~cm}^{-2}$, considering the 16 XMM-Newton

\footnotetext{
9 Observations flagged with "no" in Table 1.

10 http://xmm2.esac.esa.int/docs/documents/ CAL-TN-0018.pdf
}

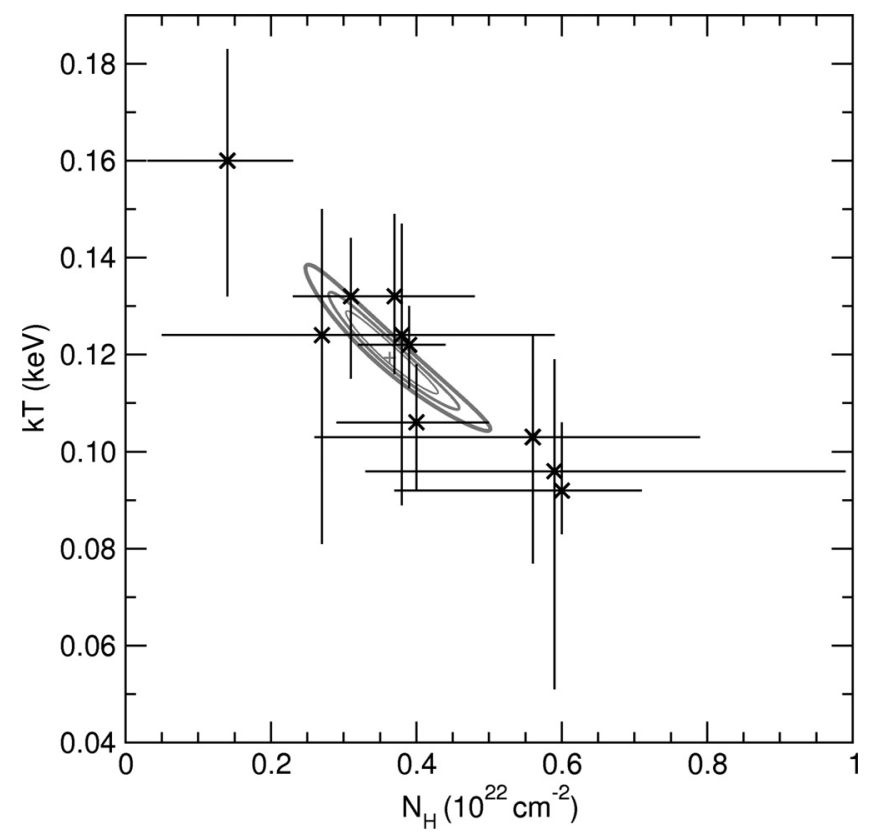

Fig. 1. Contour plot corresponding to the overall X-ray fit of source XMM J1046, using the 10 best XMM-Newton observations $(k T=$ $122_{-8}^{+10} \mathrm{eV}$ and $N_{\mathrm{H}}=3.6_{-0.7}^{+0.6} \times 10^{21} \mathrm{~cm}^{-2}$; errors are $\left.3 \sigma\right)$. The $k T-N_{\mathrm{H}}$ parameter space is shown for $1 \sigma, 2 \sigma$ and $3 \sigma$ confidence levels. The individual best fits of Table 2 are shown as crosses with $1 \sigma$ error bars.

observations; the computed range is $(7.7 \pm 0.7) \times 10^{-14}$ to $\left(12.9_{-1.6}^{+1.7}\right) \times 10^{-14} \mathrm{erg} \mathrm{s}^{-1} \mathrm{~cm}^{-2}(1 \sigma)$. Although a constant flux is not statistically acceptable $\left(\chi^{2}=43\right.$ for 15 degrees of freedom), a long term variability is excluded once the fluxes in the EPIC cameras are considered separately: the averaged flux in the pn camera is systematically fainter than that measured in the MOS cameras but both are consistent with a constant flux (at confidence levels of $\sim 19 \%$ and $\sim 92 \%$, respectively). The same $\sim 13-20 \%$ discrepancy between the two instruments is seen in the three observations where both cameras were simultaneously on. According to recent cross-calibration studies 

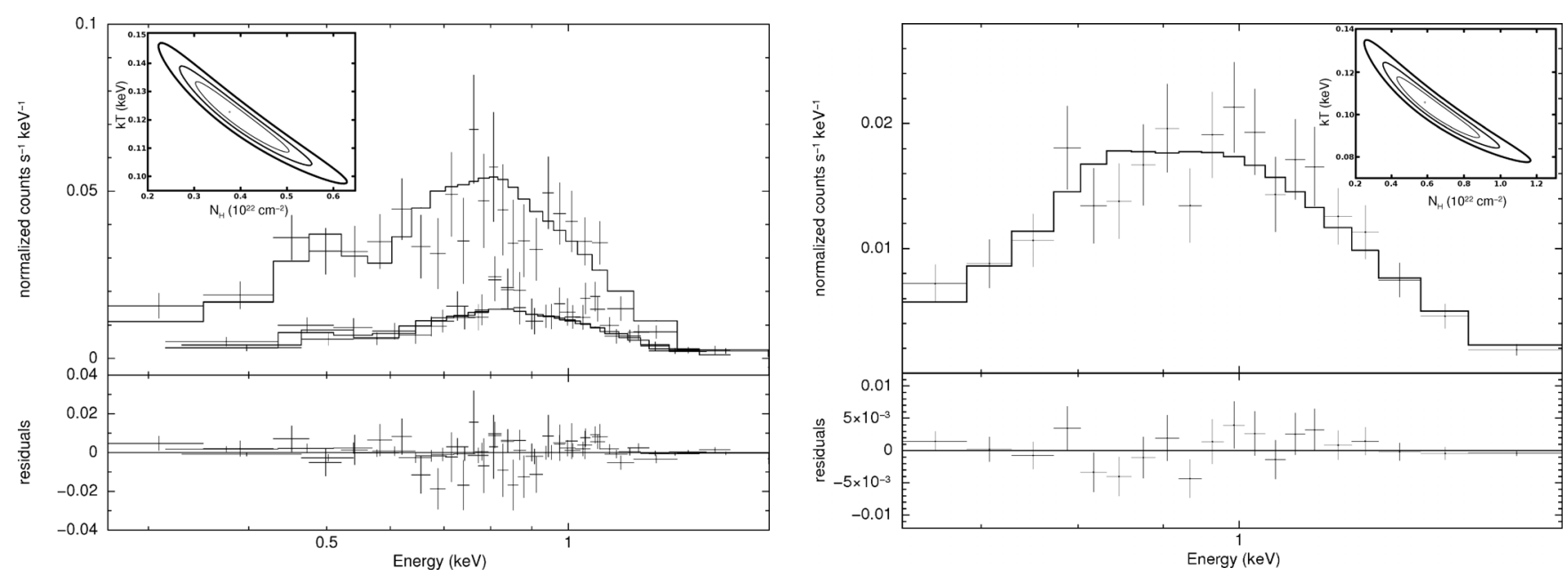

Fig. 2. X-ray spectra and best fits of source XMM J1046 as observed with XMM-Newton (left, OBSID 311990101, pn and MOS2 cameras; the two lower data points consist of two subsequent MOS2 exposures) and Chandra (right, ACIS-I). The contour plots in the insets show the $k T-N_{\mathrm{H}}$ parameter space for $1 \sigma, 2 \sigma$ and $3 \sigma$ confidence levels.

(Mateos et al. 2009), the MOS cameras register 7-9\% higher flux than pn below $4.5 \mathrm{keV}^{11}$ and this excess increases with offaxis angle. The larger discrepancy can thus be explained by the large off-axis angle of XMM J1046 in the set of analysed observations. The probabilities that temperature and column density are constant also increase when the cameras are considered individually (a consequence of the larger errors), but there is no systematic relation as for the flux (i.e. the weighted mean values are roughly the same).

Once again, accounting only for the set of 10 observations not close to a gap, the overall picture further argues against significant flux variations: the MOS and pn fluxes can be considered to be constant either when the cameras are analysed together or separately (Fig. 3). The weighted means and respective confidence levels for the two cameras are $f_{\mathrm{X}, \mathrm{pn}}=$ $(9.4 \pm 1.1) \times 10^{-14} \mathrm{erg} \mathrm{s}^{-1} \mathrm{~cm}^{-2}(44 \%)$ and $f_{\mathrm{X}, \mathrm{MOS}}=(1.07 \pm$ $0.08) \times 10^{-13} \mathrm{erg} \mathrm{s}^{-1} \mathrm{~cm}^{-2}(78 \%)$. Despite the discrepancy between the two instruments, the fluxes are compatible within errors. We adopt here the mean value of the flux as measured by the two cameras, which is also consistent with the value inferred when the cameras are analysed together, $f_{\mathrm{X}}=(1.03 \pm$ $0.06) \times 10^{-13} \mathrm{erg} \mathrm{s}^{-1} \mathrm{~cm}^{-2}$. A direct comparison of the source count rate, corrected for vignetting, for the MOS cameras is also shown in Fig. 3.

\subsection{Timing analysis}

One of the most intriguing and discernible features of the M7 is that, when compared to radio pulsars, the neutron star spin periods are longer and distributed in a much narrower range. Six of the sources show sinusoidal X-ray pulsations with pulsed fractions between $\sim 1 \%$ and $18 \%$. The detection of pulsations in the X-ray emission of source XMM J1046 would represent further evidence of its nature. However, despite the large number of archival observations that detected the source, only one (OBSID 311990101 , pn) is really suitable to conduct timing analysis - the others being either too short and having few source counts or, for the MOS observations, taken in full frame mode which has a too poor frame resolution (2.6s) for timing purposes. Similarly, the

\footnotetext{
11 The discrepancy between the cameras is even larger (of $\sim 12-13 \%$ ) at higher energies, according to the work just cited.
}

Chandra data was carried out with a $3.4 \mathrm{~s}$ time resolution imaging configuration.

With the aim to search for pulsations, we converted the photon arrival times of the pn observation from the local satellite to the solar system barycentric frame using the SAS task barycen. Source photons were extracted in a smaller elliptical region (relative to the extracted region for the spectral analysis) in order to avoid background events. For the same reason and, since XMM J1046 does not show counts above $\sim 1.5 \mathrm{keV}$, we only considered the $0.15-2 \mathrm{keV}$ energy range. We next searched for pulsations using a $Z_{n}^{2}$ (Rayleigh) test (Buccheri et al. 1983). No pulsations are found to a non-constraining $30 \%$ upper limit $(3 \sigma)$, in the $0.073-100 \mathrm{~s}$ period range; the dimness of the source would require a longer exposure in order to significantly constrain the upper limit on pulsations.

\subsection{Optical follow-up}

Follow-up optical observations performed in the $B$ and $R$ bands at the ESO Very Large Telescope (VLT) in February 2007 revealed no counterpart within $\sim 4.3 \operatorname{arcsec}(\gtrsim 5 \sigma)$ of the position of XMM J1046, $\alpha=10: 46: 08.72, \delta=-59: 43: 06.4$, as derived running the SAS task emldetect on the best XMM-Newton observation 311990101 . The $90 \%$ confidence level error circle on the position, $r_{90}=1.33$ arcsec, is given by $2.15 \sqrt{\sigma^{2}+\sigma_{\text {syst }}^{2}}$, where $\sigma$ is the nominal error as given by emldetect and $\sigma_{\text {syst }}$ is the systematic error on the detection position as provided by the $2 \mathrm{XMMp}$ catalogue, based on reliable cross-correlation with the USNO B1.0 optical catalogue. Similarly, we used the CIAO task wavdetect to determine the position and the $90 \%$ confidence level error circle using the Chandra data, which is fully consistent with the XMM-Newton results ( $\alpha=10: 46: 08.7, \delta=$ $-59: 43: 06.7, r_{90}=1.96$ arcsec).

The VLT observations consisted of short exposure $(2 \times 150 \mathrm{~s}$ in each filter) pre-imaging data which had the goal of selecting possible optical candidates for spectroscopy or deeper imaging $^{12}$. The optical images were astrometrically calibrated using non-saturated 2MASS and GSC- 2 catalogued stars and the

\footnotetext{
12 Details on the optical follow-up of this and other INS candidates are reported in a separate paper (Pires et al. 2009, submitted).
} 


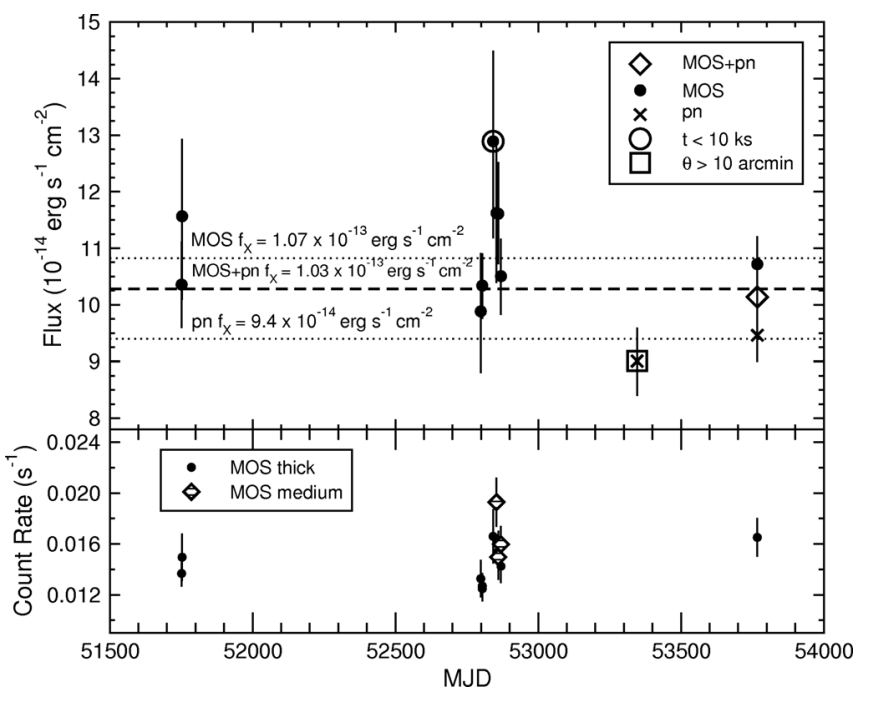

Fig. 3. Top: long term evolution of the observed flux $(0.15-3 \mathrm{keV})$ of the INS candidate XMM J1046. Only the set of $10 \mathrm{XMM}$-Newton observations not close to a gap is shown. Errors are $1 \sigma$ confidence level. The flux is stable in both pn and MOS detectors over the six-year time interval although there is a $\sim 13-20 \%$ discrepancy between the measured fluxes in these instruments. Observations with short exposure times and for which the source is located at a large off-axis angle are highlighted. Bottom: MOS on-axis count rates, discriminated by filter, for the same set of observations.

GAIA software ${ }^{13}$. We obtained additional imaging of the source at the Southern Astrophysical Research (SOAR) $4.1 \mathrm{~m}$ telescope in $\mathrm{V}$ and $\mathrm{H} \alpha(6 \times 1120 \mathrm{~s}$ and $6 \times 1575 \mathrm{~s})$ in February 2008, which also failed to detect any object. In order to compute upper limits for the optical counterpart of XMM J1046, we defined the limiting magnitude of the data as the magnitude of the faintest simulated star still succesfully measured by means of PSF fitting, on the exact location of XMM J1046. For all filters, the synthetic star was no longer detected or it was rejected (while trying to fit the PSF model) when the signal-to-noise ratio was worse than 2.5. At this confidence level (98.76\%), the present upper limits are $m_{\mathrm{B}} \gtrsim 26, m_{\mathrm{V}} \gtrsim 25.5$ and $m_{\mathrm{R}} \gtrsim 25$. Although some structure in the $\mathrm{H} \alpha$ nebular emission is present close to the position of the X-ray source, it is not clear if it is related to XMM J1046 since similar structures also appear in other parts of the nebula, see Fig. 4.

After correcting for photoelectric absorption and interstellar extinction, the above limits imply $\log \left(F_{\mathrm{X}} / F_{\mathrm{V}}\right) \gtrsim 3.1_{-0.1}^{+0.3}$. For an assumed $N_{\mathrm{H}}=(3.5 \pm 1.1) \times 10^{21} \mathrm{~cm}^{-2}, A_{V} \sim 1.96(6)$ (Predehl \& Schmitt 1995) and the unabsorbed X-ray flux ${ }^{14}$ is $F_{\mathrm{X}} \sim\left(1.4_{-0.8}^{+2.4}\right) \times 10^{-12} \mathrm{erg} \mathrm{s}^{-1} \mathrm{~cm}^{-2}$ in the $0.1-12 \mathrm{keV}$ energy band. The high value of the $\mathrm{X}$-ray-to-optical flux ratio pratically rules out any other possibility than an INS. For instance, late-type $(\mathrm{M}, \mathrm{G}, \mathrm{K})$ stars and active galactic nuclei (AGN) show logarithmic X-ray-to-optical flux ratios $\lesssim-1$ and within -1 and 1 (e.g. Barcons et al. 2007, and references therein), respectively, while cataclysmic variable (CV) systems and BL Lac objects, which are among the most extreme classes of objects, have $\log \left(F_{\mathrm{X}} / F_{\mathrm{V}}\right) \lesssim 2$ (e.g. Schwope et al. 1999). Obscured T-Tauri stars would exhibit much more absorbed X-ray spectra. We also note that the high total Galactic extinction in the

\footnotetext{
${ }^{13}$ http://star-www.dur.ac.uk/ pdraper/gaia/gaia.html

14 In this work we adopted the notation $F_{\mathrm{X}}$ and $F_{\mathrm{V}}$ to denote the unabsorbed fluxes while $f_{\mathrm{X}}$ is used for the observed flux.
}

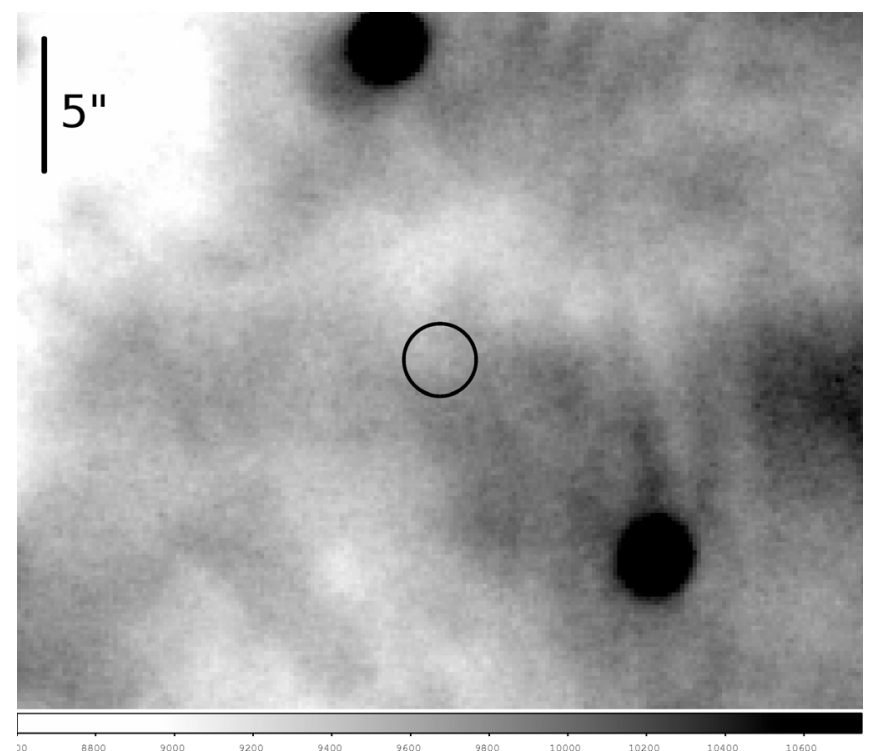

Fig. 4. Optical H $\alpha$ image of the field of XMM J1046. An inverted colour map is used, i.e. brighter objects are darker. North and east point upwards and to the left, respectively. The image was smoothed using a Gaussian function $(0.15$ arcsec). This SOAR observation is affected by fringing.

direction of XMM J1046 $(E(B-V) \sim 12$; Schlegel et al. 1998) rules out a background AGN. Moreover, the Parkes-MIT-NRAO Multibeam Survey of the southern hemisphere shows no radio source located at $\$ 6$ arcmin from the position of XMM J1046 to a flux limit of $\sim 32 \mathrm{mJy}$ (Wright et al. 1994). The Parkes Multibeam Pulsar Survey has a sensitivity of about $0.14 \mathrm{mJy}$ for a canonical pulsar (Lyne 2008; Manchester et al. 2005) and also failed to detect the source - the nearest entry in the ATNF Pulsar Catalogue is AXP 1E 1048.1-5937, at 32 arcmin from XMM J1046, and the young radio pulsar PSR J1052-5954, at $\sim 50$ arcmin.

This, together with its thermal, soft energy distribution, makes XMM J1046 a very promising INS candidate, with overall properties similar to the M7. Deep radio searches and dedicated X-ray and optical observations should be carried out in order to confirm its nature and to determine to which subgroup of INSs it may belong. For comparison, the identification of the M7 RX J2143.0+0654 with an INS was first proposed on the basis of a $~ 500$ net counts ROSAT PSPC spectrum and of follow-up optical observations yielding $\log \left(F_{\mathrm{X}} / F_{\mathrm{V}}\right) \gtrsim 3$ (Zampieri et al. 2001); the nature of the source was then confirmed by a dedicated XMM-Newton observation (Zane et al. 2005).

\section{Discussion}

On the basis of the present data, the identification of XMM J1046 with an INS appears convincing. If proven correct, this would be the first example of a presumably radio-quiet and X-ray dim INS, located at a significantly greater distance than the M7.

Most probably, all cooling INSs contained in the ROSAT Bright Source Catalogue have already been identified (Popov et al. 2000; Rutledge et al. 2003), so that new sources must be looked for at lower fluxes. The search for "blank field" sources $^{15}$ in the entire ROSAT All-Sky Survey and in HRI pointings (e.g. Agüeros et al. 2006; Chieregato et al. 2005;

\footnotetext{
15 Sources without an optical candidate in their X-ray error circles.
} 
Treves et al. 2007) produced candidates with X-ray-to-optical flux ratios of $\sim 10$ to 100 . Further X-ray and optical investigations of one such blank field led to the discovery of Calvera, a likely INS (whose exact nature is, however, still unclear) with a very large $F_{\mathrm{X}} / F_{\mathrm{V}} \gtrsim 8700$ (Rutledge et al. 2008). However, this source, like most of the other INS candidates discovered in the ROSAT data at faint fluxes, is at high Galactic latitude while it is expected that both accreting and cooling INSs at greater distances should be more abundant close to the Galactic plane.

Detailed population synthesis calculations (Posselt et al. 2008) show that, in general, cooling INSs at lower soft X-ray fluxes are also expected to be hotter than the M7 (an observational bias due to the higher photoelectric absorptions) and still at small angular distance from their birth star-forming regions. Recently, Muno et al. (2008) used 1000 XMM-Newton and Chandra archival observations covering significant part of the sky close to the Galactic plane $\left(|b| \leq 5^{\circ}\right)$ to present constraints on the number of magnetar candidates. The search was sensitive to sources with luminosities $L_{X} \geq 3 \times 10^{33} \mathrm{erg} \mathrm{s}^{-1}$ and pulsed fractions $p_{\mathrm{f}} \geq 15 \%$, at distances within a few kiloparsecs (see their Fig. 6). Interestingly, their results can also be used to constrain the number of cooling INSs, since no new pulsating neutron star candidate was found in a spin period range of $\sim 5$ to $20 \mathrm{~s}$. Rescaling luminosity down to the typical values inferred for the M7 $\left(L_{X} \geq 3 \times 10^{31} \mathrm{erg} \mathrm{s}^{-1}\right)$ we obtain that the search presented by Muno et al. is sensitive to such objects up to a few hundred parsecs. Taking into account the expected distribution of cooling INSs on the sky (Popov et al. 2005; Posselt et al. 2008), we obtain as a rough estimate that the limits by Muno et al. can be translated into $\$ 1000$ detectable cooling neutron stars up to a few hundred parsecs. In comparison with other limits this does not provide new important constraints. Still, a similar search in slightly wider period range focused on the possibility of finding cooling INSs is welcomed.

The spatial location of XMM J1046, close to the center of the Carina Nebula, and the derived value of the source column density suggest that it may be physically associated with this giant $\mathrm{H}$ II region. The Carina Nebula harbours a large number of massive stars and has ongoing active star formation (see e.g. Smith $\&$ Brooks 2007, and references therein). The source column density is a factor of $\sim 10$ higher than those typical of the M7 and is consistent with the one towards Eta Carinae $\left(N_{\mathrm{H}} \sim 3 \times 10^{21} \mathrm{~cm}^{-2}\right.$, Leutenegger et al. 2003). Eta Carinae's distance, measured with high accuracy through the expansion parallax of its circumstellar nebula, is $2.3 \mathrm{kpc}$ (e.g. Smith 2006). The distance to XMM J1046 is then likely to be comparable.

If the star surface is an isotropic blackbody emitter, then

$$
\frac{R_{\infty}}{d} \sim 3.05\left(\frac{F_{\mathrm{X}}}{10^{-12} \mathrm{erg} \mathrm{s}^{-1} \mathrm{~cm}^{-2}}\right)^{1 / 2}\left(\frac{k T}{100 \mathrm{eV}}\right)^{-2} \mathrm{~km} \mathrm{kpc}^{-1}
$$

which gives a radiation radius (as seen by an observer at infinity) of $R_{\infty} \sim 6.1 \mathrm{~km}$ for XMM J1046, assuming that it is at the same distance as Eta Carinae. Although smaller than the canonical neutron star radius, such a value is in agreement with what is measured for the M7: their redshifted radiation radii, as derived from X-ray blackbody fits and distance estimates, are in the range of $\sim 2$ to $7 \mathrm{~km}$. However, it is well known that larger emission radii (and lower temperatures) are inferred when the surface radiation is described by more realistic models which take into account the overall spectra of these sources - in particular, by invoking a geometrically thin hydrogen atmosphere on top of the condensed neutron star surface (e.g. Motch et al. 2003; Zane et al. 2004; Ho et al. 2007). Moreover, the presence of small hot regions on the surface is understood in terms of the anisotropic heat transport that occurs in the crust of cooling neutron stars endowed with a strong toroidal magnetic field component (Pérez-Azorín et al. 2006; Page et al. 2007). Recent investigations of neutron star thermal evolution which account for these effects (Aguilera et al. 2008) confirm that coolers can easily have polar caps with high temperatures and small radii.

Alternatively, the rich star-forming environment of the Carina Nebula brings the intriguing possibility that XMM J1046 could be a much older neutron star accreting from the ISM, probably born outside the nebula and whose orbit is presently intersecting the HII region. We note that, in this case, position and velocity are not expected to be correlated. The gas mass in the Carina Nebula is $\sim 10^{6} M_{\odot}$ (Smith \& Brooks 2007) which, for a typical H II region size of $\sim 100 \mathrm{pc}$, implies an average density of $\sim 10 \mathrm{~cm}^{-3}$. In fact, as reported by Mizutani et al. (2002), two distinct electron density components are detected in a $30 \mathrm{pc}$ area centered on the Carina I and II H II regions: a high-density $\left(n_{\mathrm{e}} \sim 100-350 \mathrm{~cm}^{-3}\right)$ component and an extended low-density $\left(n_{\mathrm{e}} \lesssim 100 \mathrm{~cm}^{-3}\right)$ component detectable over the entire mapped region. If XMM J1046 is moving inside the nebula, the corresponding increase in the column density is $\sim 1.5 \times 10^{20} \mathrm{~cm}^{-2} \mathrm{pc}^{-1}$, assuming a typical density of $\sim 50 \mathrm{~cm}^{-3}$. This means that the source could be up to $\sim 10 \mathrm{pc}$ inside the nebula and $N_{\mathrm{H}}$ would still be compatible with the measured value and with the one derived for Eta Carinae.

At $2.3 \mathrm{kpc}$, the luminosity of XMM J1046 is $L_{\mathrm{X}} \sim$ $\left(9_{-5}^{+15}\right) \times 10^{32} \mathrm{erg} \mathrm{s}^{-1}$. Although the estimated emission radius of XMM J1046 is comparable to those of the M7, the higher blackbody temperature is responsible for the factor of nearly 10 higher X-ray luminosity. If XMM J1046 is in the accretion phase, mass entrainment should then proceed at a rate $\dot{M}=$ $L_{\mathrm{X}} / \eta c^{2} \sim 5 \times 10^{12} \mathrm{~g} \mathrm{~s}^{-1}$, where $\eta \sim 0.2$ is the efficiency. The Bondi-Hoyle accretion rate for a star moving through the ISM with particle density $n$ is $\dot{M} \sim 10^{11} n\left(v_{10}\right)^{-3} \mathrm{~g} \mathrm{~s}^{-1}$, where $v_{10}$ is the velocity of the neutron star relative to the ISM in units of $10 \mathrm{~km} \mathrm{~s}^{-1}$. For $n \sim 10-100 \mathrm{~cm}^{-3}$, as appears likely inside the nebula, Bondi-Hoyle accretion can produce the required luminosity but the star should move very slowly through the gas, $v \sim 10 \mathrm{~km} \mathrm{~s}^{-1}$. Radio pulsars are known to have very high spatial velocity (typically $v \sim 400 \mathrm{~km} \mathrm{~s}^{-1}$; e.g. Hobbs et al. 2005; Faucher-Giguère \& Kaspi 2006), thus the chance to have an object so slow is very small. Morevover, several mechanisms are known to inhibit accretion onto a magnetized INS (Blaes et al. 1995; Toropina et al. 2003; Ikhsanov 2007).

Interestingly, and in agreement with the results of simulations by Posselt et al. (2008), a higher temperature and greater distance than those of the M7 are also observed in the only RRAT (J1819-1458, McLaughlin et al. 2007) detected up to now in X-rays. The XMM-Newton spectrum of J1819-1458 is well fitted by a blackbody with $k T \sim 140 \mathrm{eV}$ and a broad absorption feature at $\sim 1 \mathrm{keV}-$ similar to those of the M7, which are usually interpreted as evidence for magnetic fields of $\sim$ few $10^{13}-10^{14} \mathrm{G}$. Its DM (dispersion measure) distance is $\sim 3.6 \mathrm{kpc}$ and the observed flux is $f_{\mathrm{X}} \sim 1.5 \times 10^{-13} \mathrm{erg} \mathrm{s}^{-1} \mathrm{~cm}^{-2}(0.3-5 \mathrm{keV})$. The M7 and this RRAT also share similar spin periods and period derivatives. On the other hand, steady or transient radio emission are not detected in any of the M7 to a rather sensitive limiting flux of $\sim$ few $10 \mu \mathrm{Jy}$ (Kondratiev et al. 2008). In order to better understand the relations between these two classes of INSs, further, deeper pointings in X-rays and in radio are required to firmly assess the nature of this and other RRAT sources. 


\section{Summary and conclusions}

Overall, the present observational picture of XMM J1046 suggests that it has the right properties to be identified with a younger and more distant thermally emitting INS, possibly the eighth member of the ROSAT-discovered M7. The analysis of its $\mathrm{X}$-ray emission, although based on archival data obtained with non-optimal configurations, reveals an intrinsically soft energy distribution, possibly variable on long time scales. However, uncertainties on the calibration accuracy of the large off-axis angles at which the source was observed may account for the variations of the derived spectral parameters. Its mean temperature, column density and $0.15-3 \mathrm{keV}$ observed flux are $k T=117 \pm 14 \mathrm{eV}$, $N_{\mathrm{H}}=(3.5 \pm 1.1) \times 10^{21} \mathrm{~cm}^{-2}$ and $f_{\mathrm{X}}=(1.03 \pm 0.06) \times$ $10^{-13} \mathrm{erg} \mathrm{s}^{-1} \mathrm{~cm}^{-2}$, where we adopted the weighted means of the subsample of the 10 best XMM-Newton observations. The present optical limits imply a logarithmic X-ray-to-optical flux ratio greater than $\sim 3.1$, already high enough to safely exclude standard classes of X-ray emitters (late-type stars, AGN and $\mathrm{CVs}$ ). The column density is consistent with the source being located within the Carina Nebula, roughly at a distance of $\sim 2 \mathrm{kpc}$. Assuming that the X-ray emission is well described by the mean parameters given above, XMM J1046 may be more luminous and younger than the M7 and thus perhaps still close to its birth place. XMM J1046 is unique in the sense that it is hotter than the seven nearby sources and may represent an evolutionary missing link between the different classes of magnetars, radio-transient and radio-quiet INSs. The accretion scenario would require an unlikely low-velocity neutron star, combined with high accretion efficiency, to account for the X-ray luminosity of XMM J1046. A systematic search for pulsations is crucial in order to establish the true nature of this X-ray source.

Acknowledgements. The work of A.M.P. is supported by FAPESP (grant 04/04950-4), CAPES (grant BEX7812/05-7), Brazil, and the Observatory of Strasbourg (CNRS), France. R.T. aknowledges financial support from INAFASI under contract AAE TH-058. The work of S.P. is partially funded by INTAS through grant 6-1000014-5706; S.P. is also grateful to the Observatory of Cagliari for hospitality during part of this work. The authours acknowledge the use of the ATNF Pulsar Catalogue (http://www .atnf.csiro.au/research/ pulsar/psrcat). We thank the anonymous referee for useful comments and suggestions which helped to improve the paper.

\section{References}

Agüeros, M. A., Anderson, S. F., Margon, B., et al. 2006, AJ, 131, 1740 Aguilera, D. N., Pons, J. A., \& Miralles, J. A. 2008, ApJ, 673, L167 Barcons, X., Carrera, F. J., Ceballos, M. T., et al. 2007, A\&A, 476, 1191 Blaes, O., Warren, O., \& Madau, P. 1995, ApJ, 454, 370

Buccheri, R., Bennett, K., Bignami, G. F., et al. 1983, A\&A, 128, 245

Burwitz, V., Haberl, F., Neuhäuser, R., et al. 2003, A\&A, 399, 1109 Cash, W. 1979, ApJ, 228, 939

Chieregato, M., Campana, S., Treves, A., et al. 2005, A\&A, 444, 69

De Luca, A., Caraveo, P. A., Mereghetti, S., Negroni, M., \& Bignami, G. F. 2005 , ApJ, 623, 1051

Faucher-Giguère, C.-A., \& Kaspi, V. M. 2006, ApJ, 643, 332

Haberl, F. 2007, Ap\&SS, 308, 181

Haberl, F., Turolla, R., de Vries, C. P., et al. 2006, A\&A, 451, L17
Ho, W. C. G., Kaplan, D. L., Chang, P., van Adelsberg, M., \& Potekhin, A. Y. 2007, Ap\&SS, 308, 279

Hobbs, G., Lorimer, D. R., Lyne, A. G., \& Kramer, M. 2005, MNRAS, 360, 974

Hohle, M. M., Haberl, F., Vink, J., et al. 2009, A\&A, accepted [arXiv: 0810.5319]

Ikhsanov, N. R. 2007, Ap\&SS, 308, 137

Kaplan, D. L. 2008, in 40 Years of Pulsars: Millisecond Pulsars, Magnetars and More, ed. C. Bassa, Z. Wang, A. Cumming, \& V. M. Kaspi, AIP Conf. Ser., 983, 331

Kaspi, V. M., Roberts, M. S. E., \& Harding, A. K. 2004, ArXiv Astrophysics e-prints

Keane, E. F., \& Kramer, M. 2008, MNRAS, 391, 2009

Kondratiev, V. I., Burgay, M., Possenti, A., et al. 2008, in 40 Years of Pulsars: Millisecond Pulsars, Magnetars and More, ed. C. Bassa, Z. Wang, A. Cumming, \& V. M. Kaspi, AIP Conf. Ser., 983, 348

Leutenegger, M. A., Kahn, S. M., \& Ramsay, G. 2003, ApJ, 585, 1015

Lyne, A. G. 2008, in 40 Years of Pulsars: Millisecond Pulsars, Magnetars and More, ed. C. Bassa, Z. Wang, A. Cumming, \& V. M. Kaspi, AIP Conf. Ser., 983,561

Manchester, R. N., Hobbs, G. B., Teoh, A., \& Hobbs, M. 2005, AJ, 129, 1993

Mateos, S., Saxton, R. D., Read, A. M., \& Sembay, S. 2009, A\&A, 496, 879

McLaughlin, M. A., Lyne, A. G., Lorimer, D. R., et al. 2006, Nature, 439, 817

McLaughlin, M. A., Rea, N., Gaensler, B. M., et al. 2007, ApJ, 670, 1307

Mizutani, M., Onaka, T., \& Shibai, H. 2002, A\&A, 382, 610

Motch, C., Zavlin, V. E., \& Haberl, F. 2003, A\&A, 408, 323

Motch, C., Pires, A. M., Haberl, F., \& Schwope, A. 2007, Ap\&SS, 308, 217

Motch, C., Pires, A. M., Haberl, F., Schwope, A., \& Zavlin, V. E. 2009, A\&A, 497, 423

Muno, M. P., Gaensler, B. M., Nechita, A., Miller, J. M., \& Slane, P. O. 2008, ApJ, 680, 639

Ostriker, J. P., Rees, M. J., \& Silk, J. 1970, Astrophys. Lett., 6, 179

Page, D., Geppert, U., \& Küker, M. 2007, Ap\&SS, 308, 403

Pérez-Azorín, J. F., Pons, J. A., Miralles, J. A., \& Miniutti, G. 2006, A\&A, 459, 175

Pires, A. M., \& Motch, C. 2008, in 40 Years of Pulsars: Millisecond Pulsars, Magnetars and More, ed. C. Bassa, Z. Wang, A. Cumming, \& V. M. Kaspi, AIP Conf. Ser., 983, 363

Popov, S. B., Colpi, M., Treves, A., et al. 2000, ApJ, 530, 896

Popov, S. B., Colpi, M., Prokhorov, M. E., Treves, A., \& Turolla, R. 2003, A\&A, 406, 111

Popov, S. B., Turolla, R., Prokhorov, M. E., Colpi, M., \& Treves, A. 2005, Ap\&SS, 299, 117

Popov, S., Grigorian, H., Turolla, R., \& Blaschke, D. 2006, A\&A, 448, 327

Posselt, B., Popov, S. B., Haberl, F., et al. 2007, Ap\&SS, 308, 171

Posselt, B., Popov, S. B., Haberl, F., et al. 2008, A\&A, 482, 617

Predehl, P., \& Schmitt, J. H. M. M. 1995, A\&A, 293, 889

Rutledge, R. E., Fox, D. W., Bogosavljevic, M., \& Mahabal, A. 2003, ApJ, 598, 458

Rutledge, R. E., Fox, D. B., \& Shevchuk, A. H. 2008, ApJ, 672, 1137

Schlegel, D. J., Finkbeiner, D. P., \& Davis, M. 1998, ApJ, 500, 525

Schwope, A. D., Hasinger, G., Schwarz, R., Haberl, F., \& Schmidt, M. 1999, A\&A, 341, L51

Shvartsman, V. G. 1971, SvA, 14, 662

Smith, N. 2006, ApJ, 644, 1151

Smith, N., \& Brooks, K. J. 2007, MNRAS, 379, 1279

Toropina, O. D., Romanova, M. M., Toropin, Y. M., \& Lovelace, R. V. E. 2003, ApJ, 593, 472

Treves, A., Turolla, R., Zane, S., \& Colpi, M. 2000, PASP, 112, 297

Treves, A., Campana, S., Chieregato, M., et al. 2007, Ap\&SS, 308, 167 van Kerkwijk, M. H., \& Kaplan, D. L. 2007, Ap\&SS, 308, 191

van Kerkwijk, M. H., Kaplan, D. L., Pavlov, G. G., \& Mori, K. 2007, ApJ, 659, L149

Wright, A. E., Griffith, M. R., Burke, B. F., \& Ekers, R. D. 1994, ApJS, 91, 11 Zampieri, L., Campana, S., Turolla, R., et al. 2001, A\&A, 378, L5

Zane, S., Turolla, R., \& Drake, J. J. 2004, Adv. Space Res., 33, 531

Zane, S., Cropper, M., Turolla, R., et al. 2005, ApJ, 627, 397 